\title{
Large Proton Anisotropies in the 18 August 2010 Solar Particle Event
}

\author{
R.A. Leske • C.M.S. Cohen • R.A. Mewaldt • \\ E.R. Christian • A.C. Cummings • A.W. Labrador • \\ E.C. Stone • M.E. Wiedenbeck • T.T. von Rosenvinge
}

Received: 3 November 2011 / Accepted: 28 April 2012 / Published online: 6 June 2012

(C) Springer Science+Business Media B.V. 2012

\begin{abstract}
The solar particle event observed at STEREO Ahead on 18 August 2010 displayed a rich variety of behavior in the particle anisotropies. Sectored rates measured by the Low Energy Telescope (LET) on STEREO showed very large bidirectional anisotropies in 4-6 MeV protons for the first $\sim 17$ hours of the event while inside a magnetic cloud, with intensities along the field direction several hundred to nearly 1000 times greater than those perpendicular to the field. At the trailing end of the cloud, the protons became isotropic and their spectrum hardened slightly, while the $\mathrm{He} / \mathrm{H}$ abundance ratio plunged by a factor of approximately four for about four hours. Associated with the arrival of a shock on 20 August was a series of brief ( $<10$ minute duration) intensity increases (commonly called "shock spikes") with relatively narrow angular distributions ( $\left.\sim 45^{\circ} \mathrm{FWHM}\right)$, followed by an abrupt decrease in particle intensities at the shock itself and a reversal of the proton flow to a direction toward the Sun and away from the receding shock. We discuss the STEREO/LET observations of this interesting event in the context of other observations reported in the literature.
\end{abstract}

Keywords Cosmic rays, solar $\cdot$ Energetic particles, propagation $\cdot$ Magnetic fields, interplanetary

\section{Introduction}

Several significant solar energetic particle (SEP) events appeared in August 2010, which was early in Solar Cycle 24. At the location of the Ahead spacecraft of the Solar Ter-

The Sun 360

Guest Editors: Bernhard Fleck, Bernd Heber, and Angelos Vourlidas

R.A. Leske $(\varangle) \cdot$ C.M.S. Cohen · R.A. Mewaldt · A.C. Cummings · A.W. Labrador · E.C. Stone

California Institute of Technology, Mail Code 290-17, Pasadena, CA 91125, USA

e-mail: ral@srl.caltech.edu

E.R. Christian · T.T. von Rosenvinge

NASA/Goddard Space Flight Center, Greenbelt, MD 20771, USA

M.E. Wiedenbeck

Jet Propulsion Laboratory, California Institute of Technology, MS 169-327, Pasadena, CA 91109, USA 


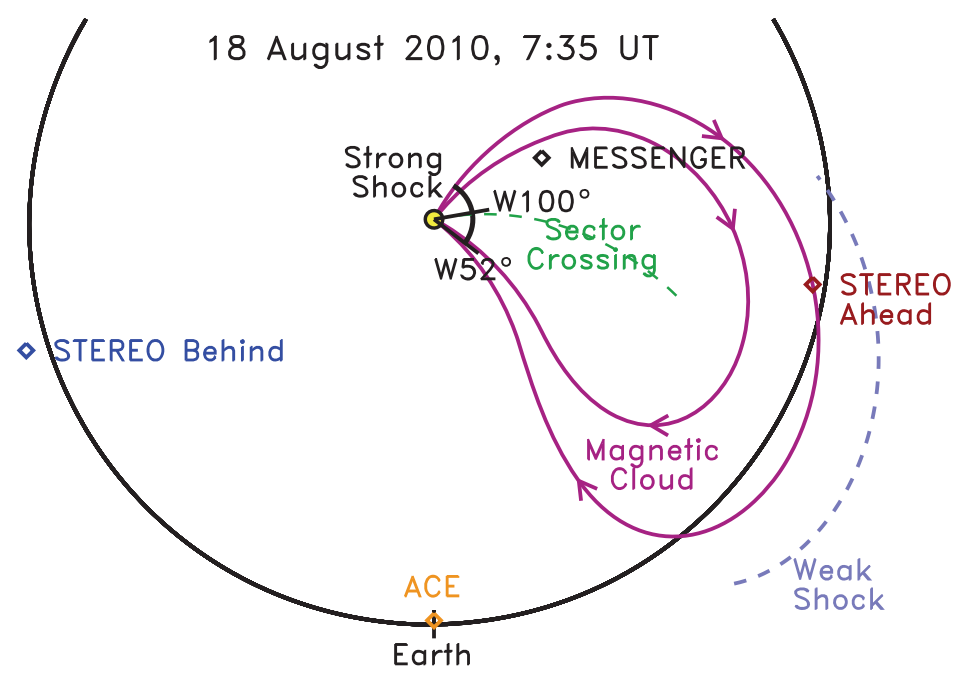

Figure 1 Schematic illustration of the positions in the ecliptic of STEREO Ahead, STEREO Behind, ACE, MESSENGER, and Earth shortly after the flare and CME of 18 August 2010 that originated from an active region located at $\sim \mathrm{W} 100^{\circ}$ at that time. Also shown are the approximate positions of a strong shock (dark blue) associated with this CME, as well as a weak shock (light blue dashed line) driven by a magnetic cloud (purple) that erupted from the same active region four days earlier when it was at $\sim \mathrm{W} 52^{\circ}$, and the change in magnetic polarity (green) between the legs of the cloud. As drawn here, the radial spacing of the features along the Sun to STEREO Ahead line is approximately correct, but the longitudinal extent and shapes of these features away from this line are purely speculative and intended for illustration purposes only.

restrial Relations Observatory (STEREO) pair ( $80^{\circ}$ west of the Earth-Sun line at the time), the event on 18 August 2010 had the highest $\sim 2 \mathrm{MeV}$ proton intensities observed between mid-December 2006 and early March 2011. Particles from this event were also detected at the Advanced Composition Explorer (ACE) and at STEREO Behind, spanning more than $150^{\circ}$ in longitude, as well as at the MESSENGER spacecraft at $\sim 0.3 \mathrm{AU}$ (http://www.srl.caltech.edu/ACE/ACENews/ACENews139.html). The locations of these spacecraft are illustrated in Figure 1. Compositionally, this event was generally typical of a large proton event, with little or no ${ }^{3} \mathrm{He}$ detected and an $\mathrm{Fe} / \mathrm{O}$ ratio (at $0.5 \mathrm{MeV} /$ nucleon) similar to that of the corona (Cohen et al., 2010). This event was associated with a C4.5 X-ray flare from active region 11099 that began on 18 August at 04:45 UT, peaked at 05:48 UT, and included type II (indicative of a shock) and type III radio bursts (http://www.swpc.noaa.gov/ftpmenu/warehouse/2010.html). The active region was over the limb at $\mathrm{N} 18^{\circ}, \sim \mathrm{W} 100^{\circ}$ at the flare onset, putting it $20^{\circ}$ west of STEREO Ahead, and the flare observed near Earth would undoubtedly have been more intense than $\mathrm{C} 4.5$ if it had not been occulted by the limb. By 05:48 UT a fast $\left(1471 \mathrm{~km} \mathrm{~s}^{-1}\right)$ partial halo coronal mass ejection (CME) was detected by SOHO/LASCO (http://cdaw.gsfc.nasa.gov/CME_list/). Region 11099 had been fairly active, producing five other C-class X-ray flares, 23 B-class flares, and at least one other CME (Steed and Lapenta, 2011) in the preceding four days. Additional details of the associated solar activity may be found in Gómez-Herrero et al. (2011).

Sectored rates measured by the Low Energy Telescope (LET; Mewaldt et al., 2008) on STEREO Ahead during this event displayed a broad variety of behavior in the particle anisotropies, ranging from isotropy to bidirectional streaming. Most striking was the very large magnitude attained by the anisotropies and their long duration, their sudden disappearance, and an interesting series of brief, highly anisotropic "shock spikes" (Sarris and Van Allen, 1974) associated with the passage of a strong shock. The observations are summarized in Table 1, and are presented in more detail below. 
Table 1 Brief chronological summary of observations at STEREO Ahead during the 18 August 2010 SEP event and sections or figures of this paper providing more detail

\begin{tabular}{|c|c|c|}
\hline Approximate time & Observed features & Section; figure \\
\hline 18 Aug 04:45-06:30 & $\begin{array}{l}\text { X-ray flare, CME, radio bursts; protons } \\
\text { released; electron event onset }\end{array}$ & Section 1; Figure 3 \\
\hline 18 Aug 06:30-07:35 & $\begin{array}{l}\text { First protons (at } E>7 \mathrm{MeV} \text { ) arrive in } \\
\text { unidirectional beam from Sun }\end{array}$ & Section 2.1 ; Figure 3 \\
\hline 18 Aug 07:35 - 13:00 & $\begin{array}{l}\text { Entry into magnetic cloud; bidirectional } \\
\text { electrons and protons }\end{array}$ & $\begin{array}{l}\text { Sections 2.2, 2.3, 3; Figures 3, } \\
4,8,12\end{array}$ \\
\hline 18 Aug 13:00 - 19 Aug 01:00 & $\begin{array}{l}\text { Unidirectional electron strahl; reduced } \\
\text { inward-flowing proton intensity; } \\
\sim 1000: 1 \text { anisotropies }\end{array}$ & $\begin{array}{l}\text { Sections 2.3, 3; Figures 5, 7, } \\
12\end{array}$ \\
\hline 19 Aug 01:00-05:00 & $\begin{array}{l}\text { Proton isotropy, broad electron strahl; } \\
\text { drop in LET He/H, dropouts in SIT }\end{array}$ & $\begin{array}{l}\text { Sections 2.3, 2.4, 3; Figures 5, } \\
8,9\end{array}$ \\
\hline 19 Aug 05:00-11:00 & $\begin{array}{l}\text { Exit magnetic cloud; narrowed electron } \\
\text { strahl; return of slight, unidirectional } \\
\text { proton anisotropy }\end{array}$ & Sections $2.3,3$; Figures $4,5,8$ \\
\hline 19 Aug 11:00-20 Aug 10:00 & $\begin{array}{l}\text { Correlated electron and proton } \\
\text { anisotropy widths and directions across } \\
\text { multiple sector boundary crossings }\end{array}$ & Section 3 ; Figures 4,8 \\
\hline 20 Aug 10:00-16:14 & $\begin{array}{l}\text { Anisotropic upstream shock spikes, } \\
\text { softer proton spectra, reduced } \mathrm{He} / \mathrm{H}\end{array}$ & Sections 2.4, 3; Figures 5, 9 \\
\hline 20 Aug 16:13:39.5 & $\begin{array}{l}\text { Strong shock, large unidirectional } \\
\text { anisotropies, hardened proton spectrum, } \\
\text { increased } \mathrm{He} / \mathrm{H} \text {, inverse velocity } \\
\text { dispersion }\end{array}$ & $\begin{array}{l}\text { Sections 2.2, 2.5, 3; Figures } 4 \text {, } \\
5,9,10,11\end{array}$ \\
\hline 20 Aug 16:14-24:00 & $\begin{array}{l}\text { Post-shock decrease in proton } \\
\text { intensities; weak, sunward-flowing } \\
\text { proton anisotropies }\end{array}$ & Sections $2.5,3$; Figures 5,8 \\
\hline
\end{tabular}

\section{Observations}

\subsection{Event Onset and Velocity Dispersion}

Time profiles of protons at energies of 1.8 to $40 \mathrm{MeV}$ measured by LET and the High Energy Telescope (HET; von Rosenvinge et al., 2008) on STEREO Ahead during the August 2010 time period are shown in Figure 2. The 18 August event occurred during an active period, but particle intensities rose about two orders of magnitude above the pre-event background. Intensities at the lower energies increased as the event progressed, peaking near a shock on 20 August, while at higher energies they peaked early in the event and fell more quickly. Such behavior is typical for large events (see, e.g., Mewaldt et al., 2005).

Velocity dispersion at the onset of the event is evident in the bottom panel of Figure 2; the higher energy protons arrived several hours before the lower energy particles. In addition to count rates binned by species, energy, and look direction, the LET instrument telemeters the pulse heights (proportional to the deposited energy) for each detector that was triggered for a sample of particle events (Mewaldt et al., 2008). Plotting the reciprocal of the velocity relative to the speed of light, $c / v$, versus the arrival time makes the velocity dispersion more obvious, and this is done for the proton pulse-height data in Figure 3. Similar $c / v$ plots have often been used to deduce the injection time of SEPs of different species relative to each other (e.g., Krucker and Lin, 2000) or relative to electromagnetic emissions from solar flares 


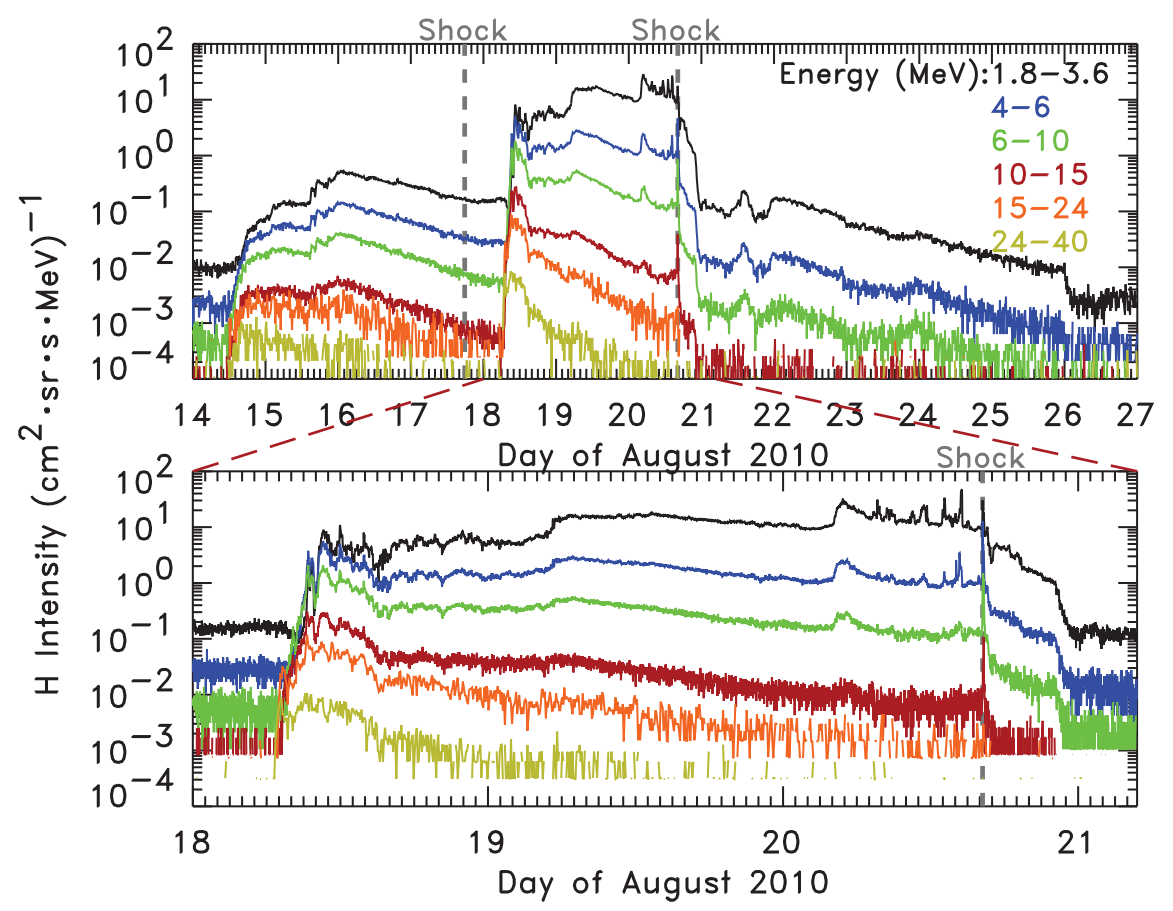

Figure 2 Time profiles of energetic protons at STEREO Ahead in six energy intervals, using data from LET (four lowest energy bands) and HET (two highest energy bands). The top panel shows the 18 August event in context with the surrounding activity, using ten-minute averages for LET and 15-minute averages for HET data. The bottom panel shows an expanded view of the 18 August event itself using one-minute averages for LET and five-minute averages for HET data. Times of shock passages (see Figure 4) are marked by vertical dashed lines.

(e.g., Lin et al., 1981; Haggerty and Roelof, 2002). However, studies have found that the implicit assumption employed in such analyses, namely that at each energy the first arriving particles traveled along the field line without scattering, may not always be warranted. If scattering is significant, the deduced pathlengths may be in error (e.g., Lintunen and Vainio, 2004; Sáiz et al., 2005; Kahler and Ragot, 2006). However, for proton energies greater than $1 \mathrm{MeV}$, simulations indicate that the inferred injection times are often roughly correct, generally with errors of only several minutes (Sáiz et al., 2005; Lintunen and Vainio, 2004).

An approximate onset edge is shown in Figure 3, from which a projected particle release time at the Sun of $\sim 05: 45$ UT is obtained. To facilitate direct comparison with the observed arrival times of electromagnetic emissions at $1 \mathrm{AU} \sim$ eight minutes must be added, bringing this to $\sim 05: 53$ UT (or conversely, the X-rays left the Sun about eight minutes earlier than observed, or at 05:40 UT, as shown in Figure 3). This release time is compatible with the solar activity discussed in Section 1, with the onset of an electron event (http://www2.physik.uni-kiel.de/stereo/downloads/sept_electron_events.pdf) observed by the Solar Electron and Proton Telescope (SEPT) on STEREO (Müller-Mellin et al., 2008), and with the value of 05:48 \pm 4 minutes (with the eight-minute time shift) reported by GómezHerrero et al. (2011).

The apparent pathlength as determined by the observed velocity dispersion is $\sim 1.7 \mathrm{AU}$, somewhat longer than the 1.2 AU length of the nominal Parker spiral field line, either because the magnetic field line was genuinely longer than nominal or because the first arriving particles had experienced significant scattering. Data from the Suprathermal Ion Telescope (SIT) on STEREO (Mason et al., 2008) extend to lower energies, below $40 \mathrm{keV} /$ nucleon (or $c / v>100$ ), and a similar pathlength is deduced from inspection of browse plots from 

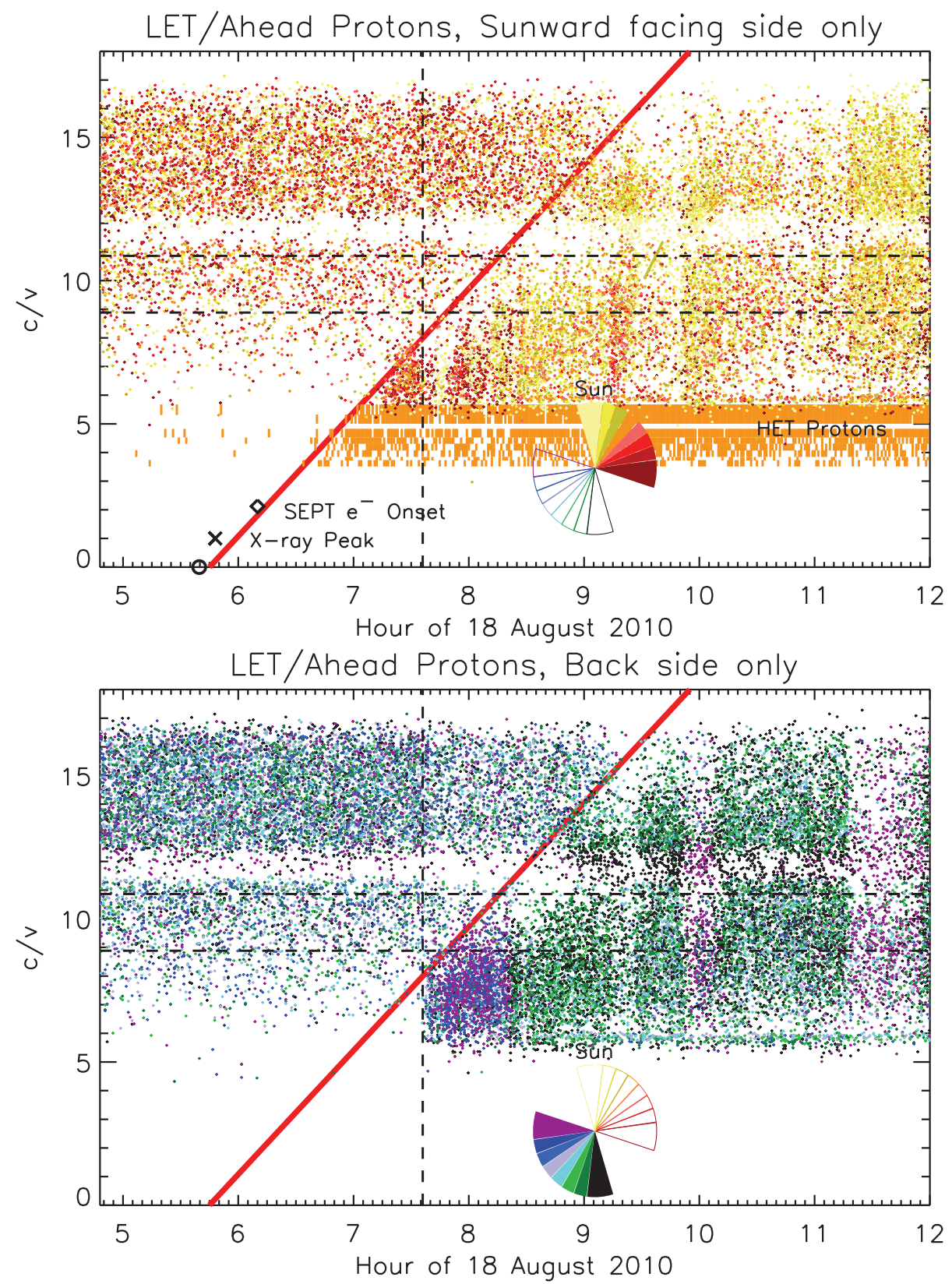

Figure 3 The reciprocal of the velocity, $v$, relative to the speed of light, $c$, plotted versus the arrival time for LET proton pulse-height events from the acceptance fan of the instrument facing toward the Sun along the nominal Parker spiral direction (top panel) and away from the Sun (bottom panel). Colors correspond to look directions in the ecliptic as indicated by the wedges in the pie chart legends. Also indicated in the top panel in the $c / v$ range of $\sim 3-5.5$ are periods when the HET proton rates in eight energy bands were nonzero (orange, corresponding to the HET viewing direction), the onset of an electron event observed by SEPT (diamond), the observed peak time of the X-ray flare $(x)$, and the time when the peak X-ray emission would have left the Sun (circle). The diagonal line, which is the same in each panel, marks the approximate onset of the event based on the higher energy LET and HET data and corresponds to a pathlength of $\sim 1.7$ AU. Horizontal dashed lines bracket the range of $c / v$ values corresponding to $4-6 \mathrm{MeV}$ protons, for which sectored rate data are available, while the vertical dashed line marks the start of a magnetic cloud (see Figure 4). Vertical banding is largely due to changes in the magnetic field direction. Horizontal gaps near $c / v=11-12$ and $\sim 4.9$ arise from decreased detection efficiency in LET and HET, respectively, for protons that just penetrate the third detector in each instrument stack without depositing enough energy to trigger its relatively high threshold (Mewaldt et al., 2008; von Rosenvinge et al., 2008). 
this instrument (http://www.srl.caltech.edu/STEREO/Level1/SIT_public.html). At the higher energies $(c / v<8)$ shown in Figure 3, note that particles arrived from the sunward direction up to an hour before they were detected coming from the anti-Sun direction. As we discuss more in the next section, the arrival of particles from the anti-Sun direction coincided with the entrance of the spacecraft into a magnetic cloud. Although the onset edge became less distinct inside the cloud, velocity dispersion was still clearly present from both the sunward and anti-Sun directions. For example, the increase in intensities near $c / v \sim 15$ first occurred more than an hour after cloud entry, while at higher energies $(c / v<8)$ the intensities were elevated immediately upon entry. The blurriness of the onset edge precludes any definitive determination as to whether or not the pathlength inside the cloud differed significantly from that outside the cloud; some studies suggest that there should be no difference (e.g., Kahler, Haggerty, and Richardson, 2011).

\subsection{Plasma Parameters: Shocks and Magnetic Cloud}

Figure 4 shows magnetic field and solar wind parameters during this event, obtained from the magnetometer instrument, MAG (Acuña et al., 2008), and the Plasma and Suprathermal Ion Composition (PLASTIC) instrument (Galvin et al., 2008) on STEREO Ahead. At 09:38 UT on 14 August, a C4.4 X-ray flare erupted from active region 11093 or 11099 (the region later associated with the 18 August flare) or both (http://www.spaceweather.com/ archive. php?view=1\&day=14\&month=08\&year=2010); the centers of the two regions were within $7^{\circ}$ of heliographic latitude and longitude of each other, making it difficult to assign activity to one region alone (http://www.swpc.noaa.gov/ftpmenu/warehouse/2010.html). A halo CME associated with this event, with the position angle of the fastest moving segment of the CME edge directed westward (toward STEREO Ahead) at $224^{\circ}$, was observed by SOHO/LASCO by 10:12 UT, with a plane-of-sky speed of $1205 \mathrm{~km} \mathrm{~s}^{-1}$ that had fallen to $989 \mathrm{~km} \mathrm{~s}^{-1}$ by 20 solar radii (http://cdaw.gsfc.nasa.gov/CME_list/). This earlier activity was responsible for the particle increase observed on 14 August in the upper panel of Figure 2.

As seen in Figure 4, around 17:50 UT on 17 August, a weak shock passed the spacecraft (Steed and Lapenta, 2011), driven by the interplanetary coronal mass ejection (ICME) associated with the 14 August flare. Based on the transit time of the shock, the ICME must have undergone further deceleration between 20 solar radii and 1 AU. From about 07:35 UT on 18 August until 05:11 UT on 19 August, the spacecraft was inside a magnetic cloud from this ICME (K. Steed, private communication). The cloud boundaries were determined using a minimum variance analysis technique (Sonnerup and Cahill, 1967) to rotate the measured magnetic field components to the local magnetic cloud coordinate system in order to separate the azimuthal and axial components and select the interval that showed the clearest rotation, as in Steed et al. (2011). Characteristics of such clouds (Burlaga et al., 1981) that appear in Figure 4 include a clear rotation of the magnetic field latitude, accompanied by an increase (albeit a small one, only $\sim 1 \mathrm{nT}$ ) in the field magnitude, with proton temperatures lower than expected in normal solar wind during at least part of this period (indicated by the blue dashed line in Figure 4 from the formula of Elliott et al., 2005). In addition, as we discuss later, bidirectional suprathermal electrons were present for the first $\sim 5$ hours of the cloud (see Figure 8 ). Throughout most of 18 and 19 August, the magnetic field longitude was displaced from the nominal Parker spiral direction by $\sim 50-60^{\circ}$. A strong shock arrived at Ahead at 16:13:39.5 UT on $20 \mathrm{Au}-$ gust, with a magnetosonic Mach number of 4 and a shock normal angle of $\theta_{\mathrm{Bn}}=57^{\circ}$ (http://www-ssc.igpp.ucla.edu/ jlan/STEREO/Level3/STEREO_Level3_Shock.pdf), driven by another ICME most likely corresponding to the CME observed by SOHO/LASCO 


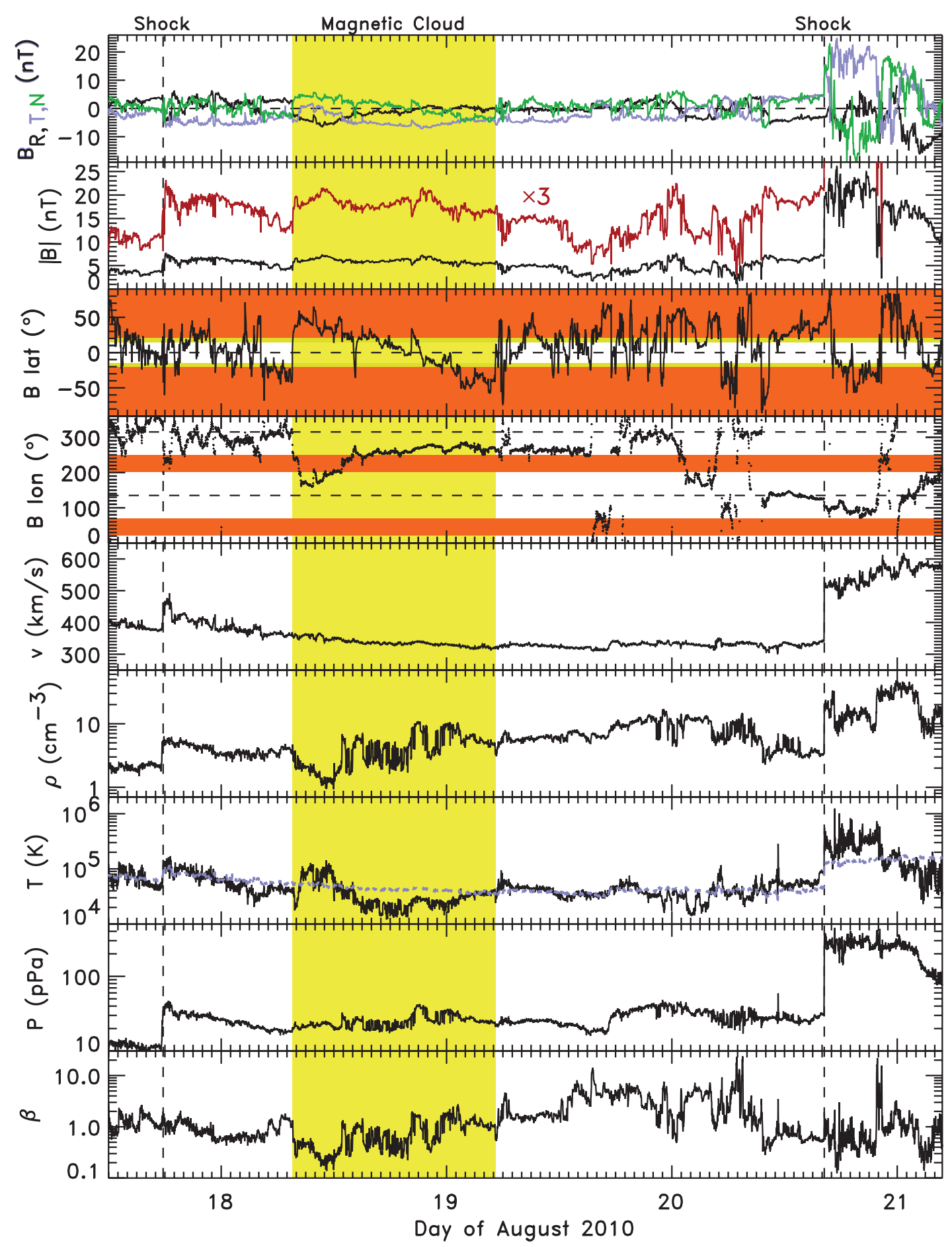

Figure 4 Plasma parameters at STEREO Ahead using one-minute resolution data. From top to bottom, panels show the components of the magnetic field in Radial-Tangential-Normal (RTN) coordinates, the magnitude, RTN latitude (i.e., $\left.\sin ^{-1}\left(B_{\mathrm{N}} /|B|\right)\right)$ and longitude $\left(\tan ^{-1}\left(B_{\mathrm{T}} / B_{\mathrm{R}}\right)\right)$ of the field, the speed, density, and temperature of solar wind protons, the total (magnetic plus plasma thermal) solar wind pressure, and the plasma beta. The top four panels are from MAG; the bottom five incorporate data from PLASTIC. The red trace in the $B$ magnitude panel is a factor of three enlargement to better show the small enhancement in the magnetic cloud highlighted in yellow. In the $B$ longitude and latitude panels, red shaded regions indicate directions outside of LET's field of view, while horizontal dashed lines mark the nominal Parker spiral field direction. The dotted blue line in the temperature panel is the expected temperature at the given solar wind speed from the (rarefaction) formula of Elliott et al. (2005). Two shocks are indicated by vertical dashed lines. 
on 18 August (http://www-ssc.igpp.ucla.edu/ jlan/STEREO/Level3/STEREO_Level3_ICME. pdf). Most of the energetic protons observed by LET and HET during this event were presumably accelerated by this shock when it was closer to the Sun.

\subsection{Particle Anisotropies}

The LET instrument measures sectored rates in 16 different viewing directions, distributed in two fans each spanning $133^{\circ}$ of longitude in the ecliptic and $\sim 30-40^{\circ}$ of latitude out of the ecliptic, one pointing toward the Sun centered along the nominal Parker spiral direction and the other looking in the anti-Sun nominal field direction (Mewaldt et al., 2008). In August 2010, sectored rates for protons were measured in only a single energy band of 4-6 MeV (in late November 2010 the instrument was reconfigured to also provide proton sectored rates at $1.8-3.6 \mathrm{MeV}$ and $6-10 \mathrm{MeV}$ ); time profiles of these sectored proton intensities are shown in Figure 5. By the time 4-6 MeV protons from the SEP event arrived, the Ahead spacecraft was already inside the magnetic cloud (Figure 3); thus LET does not provide detailed anisotropy information about the event onset before the cloud. At the Behind spacecraft (from which the solar source of the activity was $\sim 80^{\circ}$ beyond the west limb), fairly typical behavior was observed, with a period of moderate (factor of approximately ten), unidirectional anisotropies corresponding to particle flows away from the Sun along the magnetic field for several hours at the onset of the event. However, at STEREO Ahead, a remarkable, extended period of very large bidirectional anisotropies occurred during the first day of the event; ratios of observed maximum to minimum sectored intensities reached as high as $\sim 1000$. Early in the event the time profiles for different sectors repeatedly cross each other as the magnetic field line wandered in longitude from sector to sector, as seen in Figure 6 where the anisotropies and field direction are directly compared. The apparent reduction in anisotropies on 18 August between 12:00 UT and 15:00 UT in Figure 5 occurred when the $B$ field and particle beam swept through a gap in the LET field of view (Figure 6) and thus the full magnitude of the anisotropies went undetected. Large anisotropies persisted for more than 17 hours, from the event onset (at these energies) before 08:00 UT on 18 August until 01:00 UT on 19 August.

For the last half of the day on 18 August the field direction was relatively steady. Figure 7 shows the angular distribution of protons (in the spacecraft frame) obtained by averaging over more than five hours during this period, compared with the $B$ field direction. The alignment of the particle beam with the field may not be entirely clear, since the $B$ field was near coverage gaps in the LET field of view and the sectors adjacent to the gaps are twice as wide as the others. It is perhaps more obvious that the directions of minimum intensities were orthogonal to the field direction. Even when averaged over such a long time, the observed maximum to minimum sectored intensity ratio is nearly 100 . The particle flow was clearly bidirectional at this time (see also Figure 6), with intensities coming from the Sun roughly five times higher than those flowing back toward the Sun.

By using the measured field directions from MAG on STEREO (Acuña et al., 2008) together with the modeled response of the LET instrument in each viewing direction, and correcting the LET 4-6 MeV proton sectored intensities and view directions from the spacecraft frame to the solar wind frame by accounting for the Compton-Getting anisotropy (Ipavich, 1974), true pitch angle distributions were calculated. The temporal evolution of the proton pitch angle distributions is shown in Figure 8, where they are compared with pitch angle distributions of the phase space densities of suprathermal electrons at $194-314 \mathrm{eV}$ measured by the Solar Wind Electron Analyzer (SWEA) on STEREO (Sauvaud et al., 2008) 


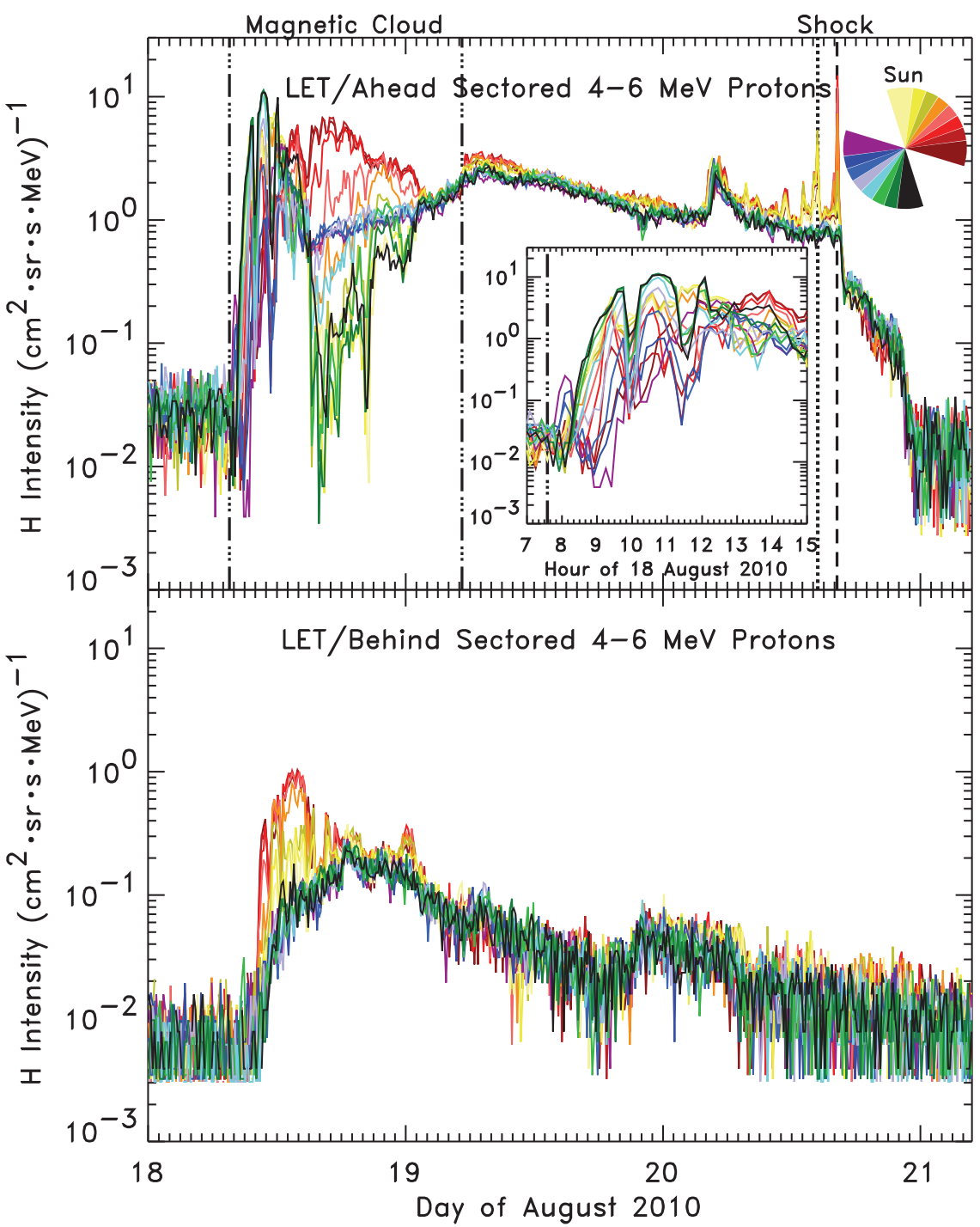

Figure 5 Time profiles of ten-minute averaged 4-6 MeV protons from LET on STEREO Ahead (top panel) and Behind (bottom panel) in 16 different sectors, with intensities adjusted to the solar wind frame by correcting for the Compton-Getting anisotropy (Ipavich, 1974). Viewing directions in the ecliptic and sector widths for each of the 16 colored traces are represented by wedges with the corresponding color in the pie chart inset. Passage of a strong shock is marked by a dashed line and the largest upstream shock spike by a dotted line, while the boundaries of the magnetic cloud from Figure 4 are indicated by dot-dashed lines. The inset in the upper panel shows an expanded view near the event onset. Error bars have been omitted for clarity throughout. During the magnetic cloud interval in the upper panel the counts per sector range from $<5$ in the minimum intensity directions to $>3000$ counts per 10 minute period in the peak intensity directions.

obtained from http://stereo.cesr.fr. The widths and flow directions of the proton anisotropies show a good correlation with those of the suprathermal electrons, as we discuss more in Section 3.

From about 01:00 UT to 05:00 UT on 19 August, at the trailing end of the magnetic cloud, the protons became nearly isotropic, as seen in Figure 5. Just outside the cloud there was a small $(\sim 30 \%)$ excess of particles coming from the sunward direction that lasted for about six hours, disappeared, and returned by the end of 19 August. In our preliminary report (Leske et al., 2011), before removing the Compton-Getting anisotropy, this excess appeared larger and more persistent. Upstream of the strong shock on 20 August, a series of 


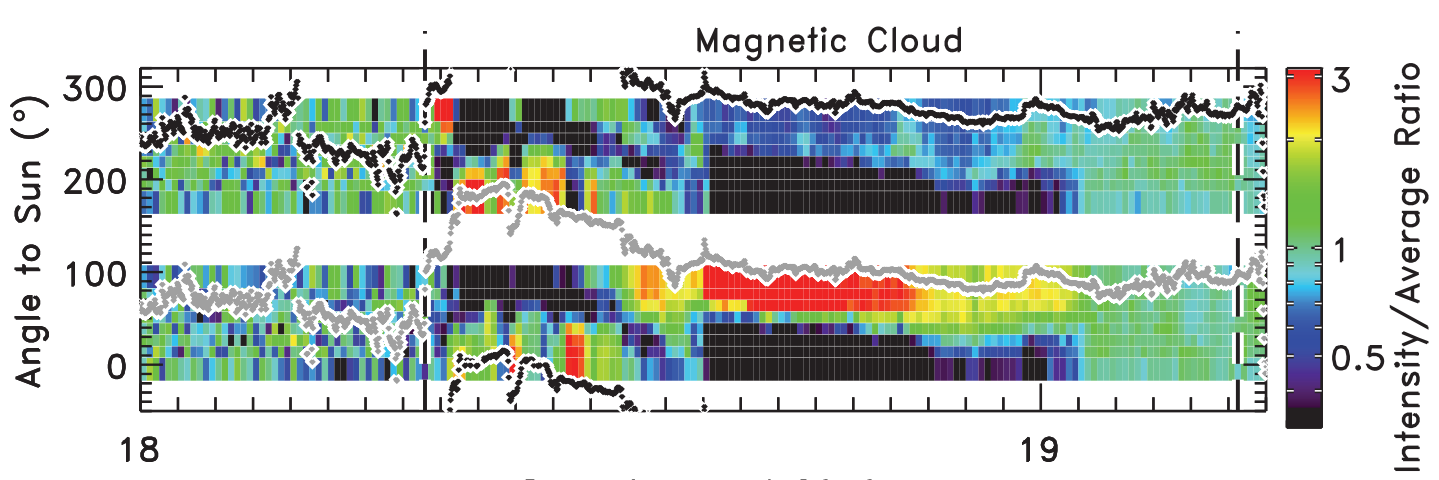

Day of August 2010

Figure 6 Ratio of the 4-6 MeV proton intensity in each of 16 sectors to the sector-averaged intensity (in ten-minute intervals) from LET on STEREO Ahead is shown (color scale); white bands are outside the LET field of view. Superposed are the directions both parallel (black symbols) and antiparallel (gray symbols) to the one-minute averaged magnetic field longitudes; the boundaries of the magnetic cloud in Figure 4 are marked by dashed lines. Although the color scale saturates at ratios of $\sim 0.3$ and 3 , proton anisotropies were at times much larger than this (see Figure 5).

Figure 7 Polar plot (on a logarithmic radial scale) of the directional intensities of 4-6 MeV protons in the R-T plane (approximately the ecliptic) averaged over the time interval of 14:53 UT to $20: 24$ UT on 18 August; gray lines mark the projected magnetic field direction using all one-minute samples during this period.

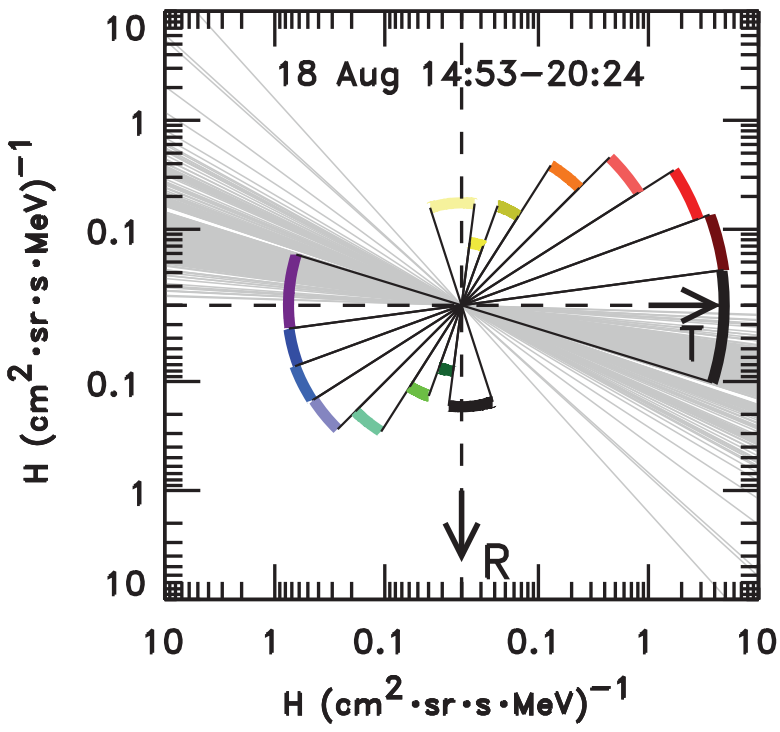

short duration (about ten minutes) highly anisotropic shock spikes appeared, similar to those observed in the vicinity of shocks in previous studies (e.g., Sarris and Van Allen, 1974).

\subsection{Evolution of Spectra and Composition}

The intensity ratio of the $4-6 \mathrm{MeV}$ to $1.8-3.6 \mathrm{MeV}$ protons shown in Figure 9 provides an estimate of the hardness of the proton energy spectrum and tracks its evolution throughout the event. The large velocity dispersion at the onset (Figures 2 and 3) resulted in this ratio reaching a maximum of $\sim 3$, equivalent to a spectral index of +2 ; the ratio exceeded unity for $\sim 1.5$ hours. The spectral hardness decreased more or less steadily during the event, dropped at the upstream shock spikes, and increased at the shock passage on 20 August. The $\mathrm{He} / \mathrm{H}$ ratio at $1.8-3.6 \mathrm{MeV} /$ nucleon in the bottom panel of Figure 9 averaged about 0.004 throughout the event, about an order of magnitude lower than the average value in large SEP events of $0.0363 \pm 0.0054$ at $1-4 \mathrm{MeV} /$ nucleon (Reames, 1995). At slightly higher energies of $4-6 \mathrm{MeV} /$ nucleon the $\mathrm{He} / \mathrm{H}$ ratio was substantially lower still, by a factor of $\sim 2-3$. Such 


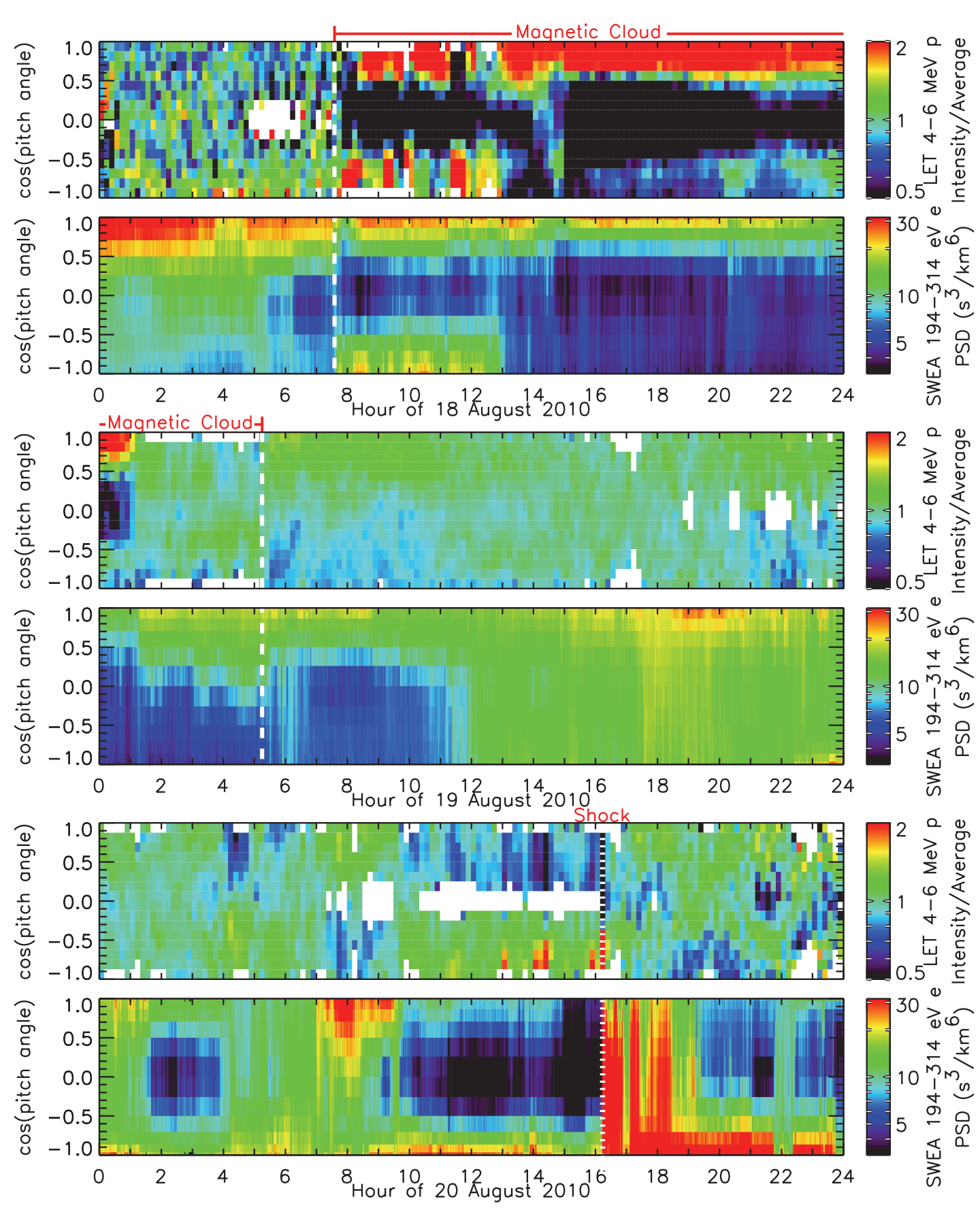

Figure 8 Pitch angle distributions of the intensity ratio of 4-6 MeV protons to the sector-averaged intensity in each of 16 bins, corrected for the Compton-Getting anisotropy, in ten-minute intervals from LET on STEREO Ahead (top panels of each pair); white areas indicate directions outside the LET field of view. For comparison, the bottom panels of each pair show the pitch angle distribution of the phase space density (PSD) of suprathermal electrons (at 194-314 eV) from SWEA on STEREO. The color scale for the LET panels was chosen to accentuate the subtle anisotropy variations on 19 and 20 August and thus is heavily saturated in the 18 August panel. Dashed lines mark the magnetic cloud boundaries on 18 and 19 August, while a dotted line indicates the time of the shock passage on 20 August.

a large drop in $\mathrm{He} / \mathrm{H}$ over this small energy interval is apparently not uncommon (Mewaldt et al., 2005). Although nearly constant during most of the event after the magnetic cloud, the $\mathrm{He} / \mathrm{H}$ ratio decreased at the upstream shock spikes, but actually increased at the shock itself by more than a factor of two at low energies. 


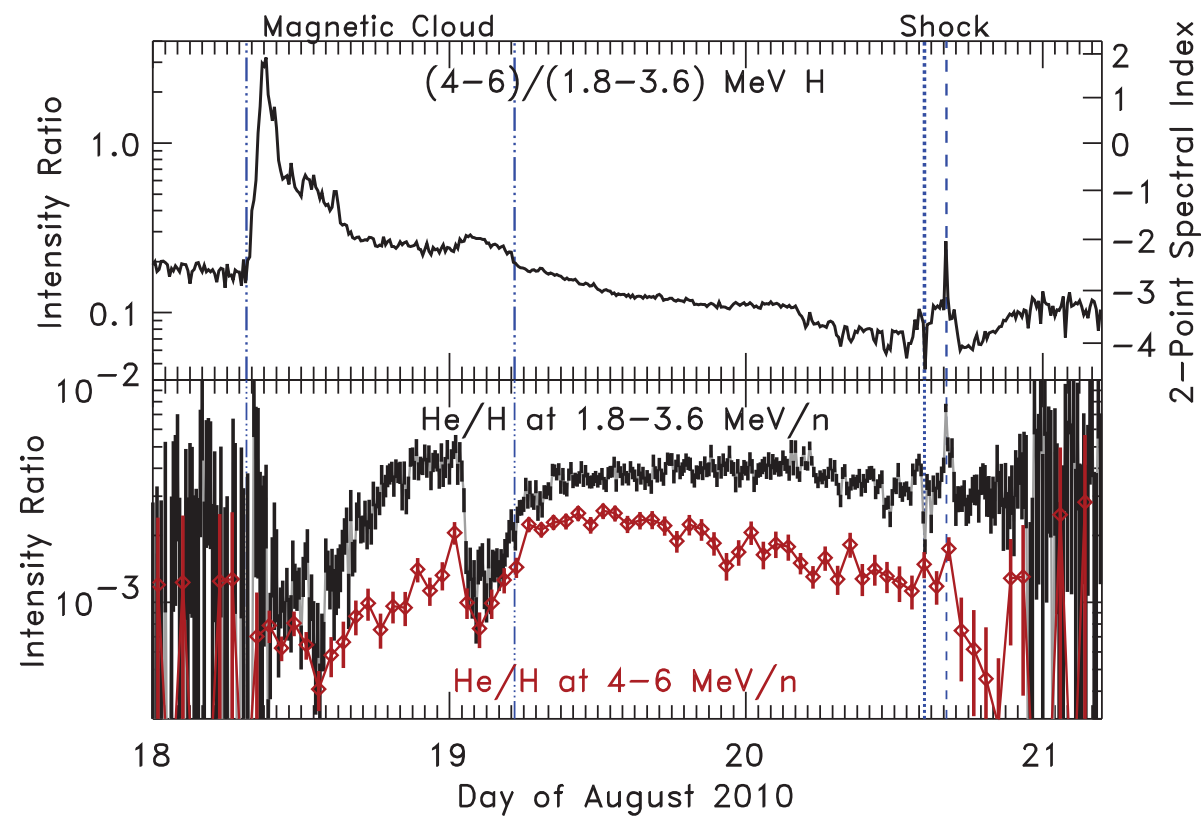

Figure 9 Intensity ratio of the 4-6 MeV to 1.8-3.6 MeV protons from LET on STEREO Ahead (top panel) and the corresponding spectral index (right axis). The bottom panel shows the He/H ratio at 1.8-3.6 MeV/nucleon (black) and at 4-6 MeV/nucleon (red diamonds), each with statistical uncertainties. Ten-minute averages are used throughout except for the $4-6 \mathrm{MeV} /$ nucleon $\mathrm{He} / \mathrm{H}$ ratio, where hour averages are used. The time of the shock passage is marked with a dashed line and that of the largest of the upstream shock spikes with a dotted line (see Figure 5), while the magnetic cloud boundaries are indicated by the dot-dashed lines.

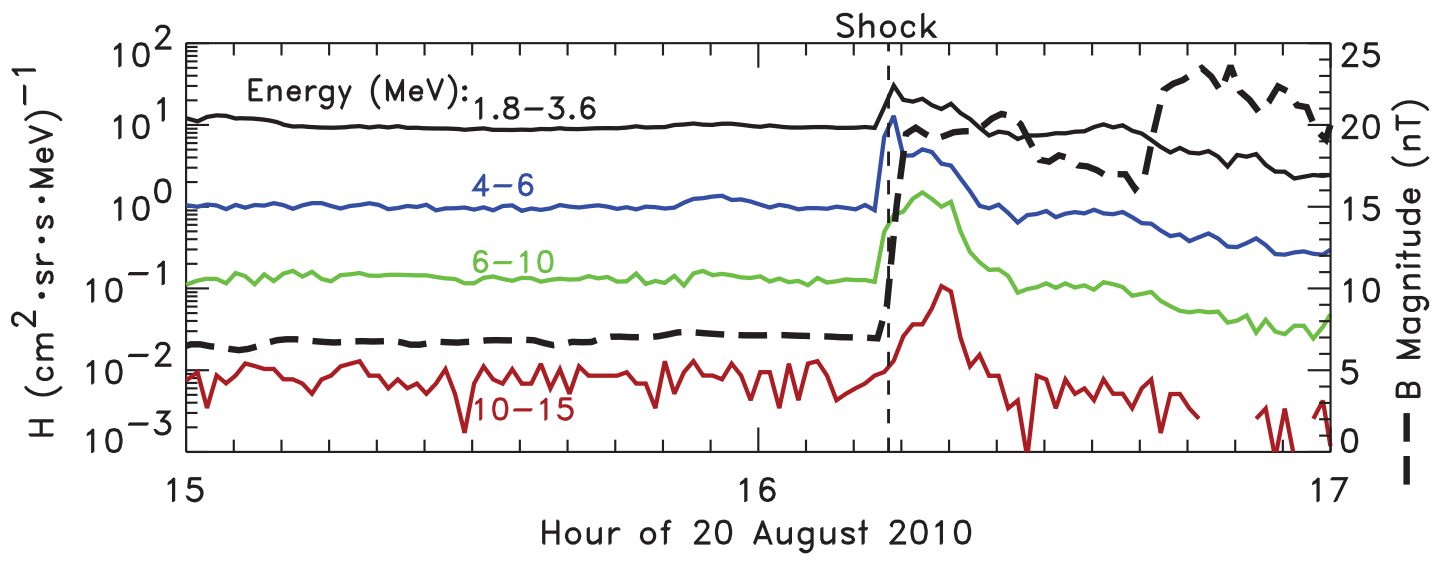

Figure 10 One-minute averaged proton rates from LET on STEREO Ahead (at the energies indicated) near the shock spike of 20 August 2010, compared with the magnetic field magnitude (thick dashed line; right axis). The shock is marked with a vertical dashed line.

\subsection{Behavior at the Shock}

At higher time resolution, the intensity peak at the shock spike on 20 August clearly occurs at different times for different energies, as shown in Figure 10. At the lowest energies shown, the peak occurs within about one minute after the shock passage, but about five minutes after the shock at the higher energies. Although typically such spikes do not extend beyond $\sim 5 \mathrm{MeV}$ (Sarris and Van Allen, 1974), this spike is clearly visible even at $10-15 \mathrm{MeV}$. The protons were narrowly beamed $\left(\sim 45^{\circ} \mathrm{FWHM}\right)$ unidirectionally along the field during 
Figure 11 Polar plot of the in-ecliptic directional intensities (with statistical uncertainties) in 16 sectors of 4-6 MeV protons from LET on STEREO Ahead averaged over a three-minute period at the shock spike of 20 August 2010. Gray lines show the three one-minute averaged magnetic field directions during this interval.

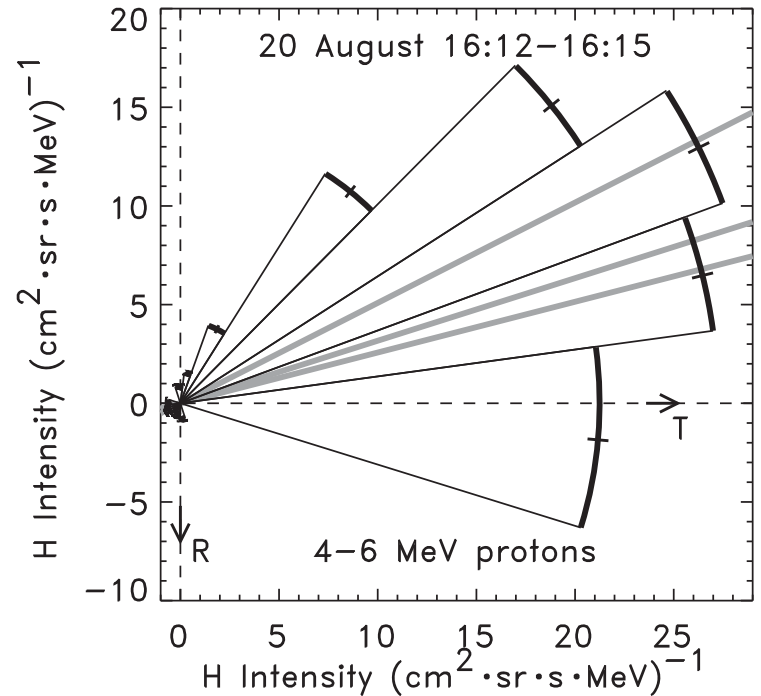

the shock spike (Figure 11). Shortly downstream of the shock the intensities dropped precipitously and became nearly isotropic (Figure 5), as is often observed; the small anisotropy that was present had reversed direction relative to the suprathermal electrons and was now flowing back toward the Sun from the shock (Figure 8).

\section{Discussion}

The field and plasma observations (Figure 4) indicate that STEREO Ahead was in the sheath region between a weak shock and a magnetic cloud that originated from an area comprising active regions 11093 and 11099 (Steed and Lapenta, 2011) when the flare on 18 August (and subsequent CME and shock) erupted from the same region. Particles initially arrived only from the sunward direction (Figure 3). At the particle onset, the magnetic field was close to the nominal Parker spiral direction (Figure 4), but the pathlength to the particle source was longer by $\sim 0.5 \mathrm{AU}$, probably due to disturbance of the field in the sheath or deflection of the field lines around the oncoming cloud. The moment the spacecraft entered the magnetic cloud, bidirectional proton streaming was observed (Figure 3). Such bidirectional flows may arise if particles are injected at both footpoints of the magnetic cloud or if they undergo magnetic mirroring.

The component of the solar wind suprathermal electron heat flux that is magnetically focused into a beam, or strahl, provides a useful diagnostic of the magnetic field topology, with the direction of the strahl indicating the direction of the field line pointing outward, away from the Sun (Gosling et al., 1987; Crooker et al., 2004). Beamed suprathermal electron flow both parallel and antiparallel to the field at comparable intensities suggests a closed field line topology, with both ends rooted in the photosphere. Such an interval is evident in the pitch angle distributions measured by SWEA on 18 August from $\sim$ 07:30 - 13:00 UT (Figure 8). Although the heat flux is generally bidirectional during this period, it appears somewhat intermittent (as does the proton bidirectional streaming in LET); this behavior has been observed before and attributed to patchy disconnection of one or both ends of the magnetic cloud field lines from the Sun (Larson et al., 1997). After 13:00 UT, while still inside the magnetic cloud (based on the field rotation in Figure 4), the electron strahl became persistently unidirectional, suggesting that the spacecraft had moved onto open field lines and that one end of the magnetic cloud had disconnected from the Sun. At the same time, 
Figure 12 Examples of pitch angle distributions of $4-6 \mathrm{MeV}$ protons in two ten-minute time intervals within the magnetic cloud of 18 August 2010, one in the closed field line region (blue diamonds; see Figure 8) and the other in the open field region (red squares).

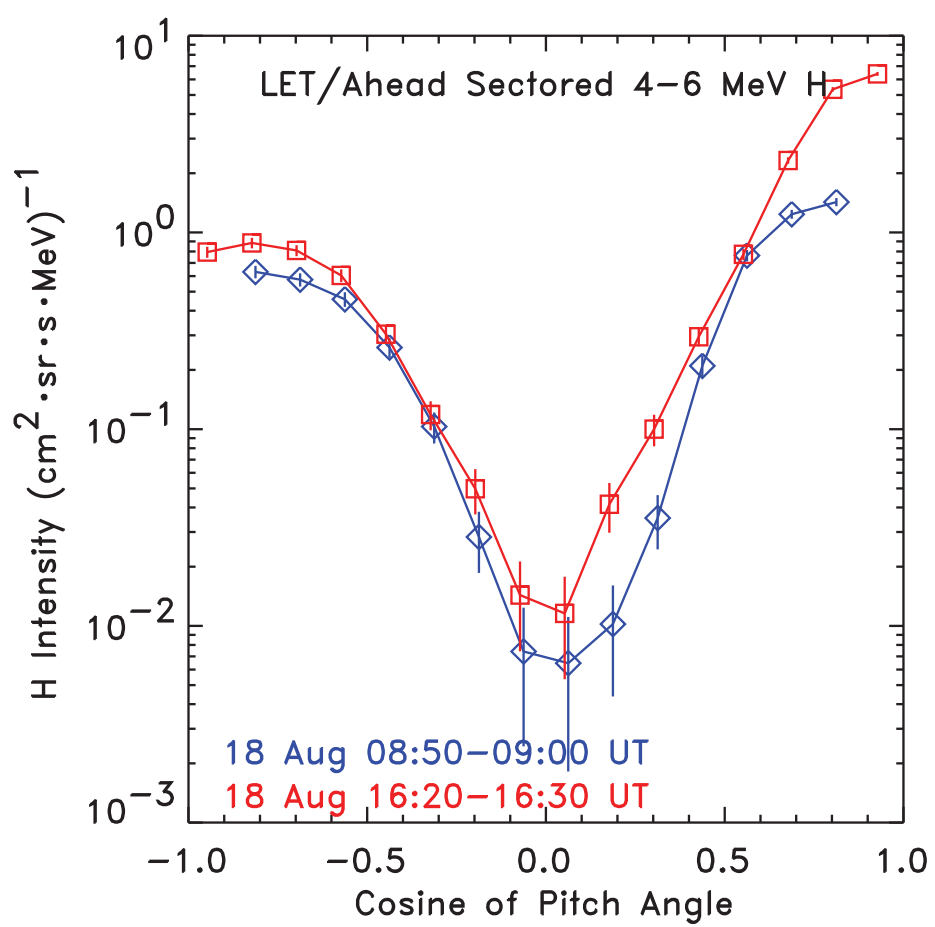

the LET proton anisotropies were still bidirectional but exhibited a distinct change. Instead of roughly equal intensities flowing in both directions, as had been seen in the closed field region, a much higher intensity (by a factor of $\sim 5-10$ ) flowed in the same direction as the strahl than in the opposite direction.

The difference in the proton pitch angle distributions between the closed field region and open field region is illustrated in Figure 12. The lower intensity peaks at pitch angle cosines near -1 appear significantly broader than the higher intensity peaks. This suggests that there was relatively more scattering (or less focusing) in one leg of the cloud than the other, but whether this was due to a difference in turbulence in the two legs or simply a difference in the lengths of the legs is not clear without modeling and further analysis. The steep shapes of these pitch angle distributions, with drops in intensity of nearly 1000 between directions aligned with the field and perpendicular to it, are very similar to those observed by the Energetic and Relativistic Nuclei and Electron (ERNE) instrument on SOHO in the 2 May 1998 SEP event (Torsti, Riihonen, and Kocharov, 2004). The May 1998 event originated from an active region $15^{\circ}$ west of the spacecraft only 1.5 hours after a magnetic cloud from the same region reached SOHO, much like the situation encountered by STEREO Ahead in the 18 August 2010 event. Calculations by Torsti, Riihonen, and Kocharov (2004) showed that a parallel mean free path of at least $10 \mathrm{AU}$ was required to produce such large anisotropies and steep pitch angle distributions.

Although the bidirectional protons in the closed field region may be the result of magnetic mirroring, the similar behavior of the suprathermal electron and energetic proton anisotropies suggests that prior to $\sim 13: 00$ UT on 18 August, protons were being released at both footpoints of the magnetic cloud. This could happen if extended injection (lasting $>7$ hours) occurred at the Sun over a region large enough to encompass both footpoints, but although relatively long lasting, the duration of the X-ray flare itself was only about two hours (http://www.swpc.noaa.gov/ftpmenu/warehouse/2010.html; Gómez-Herrero et al., 2011). Alternatively, the outward moving shock that arrived on 20 August, which must have overtaken the preceding magnetic cloud, may have intersected with, and ac- 
celerated particles into, both legs of the cloud, as shown in the sketch of Figure 1. However, inside the open field region after 13:00 UT, the situation is less clear. If the open field lines encountered a compression region, magnetic mirroring may again account for the counterstreaming proton flow. Alternatively, the open field (if it were still configured similarly to a flux rope, that is, curved back toward the Sun) might have been in contact with the shock at a location with a field geometry or shock strength changed sufficiently to affect the shock acceleration rate or efficiency (Giacalone and Kóta, 2006; Giacalone and Jokipii, 2006), or the seed particle density in that region of the shock may have dropped enough to reduce the energetic proton intensity coming from the disconnected leg of the cloud.

Therefore, it is not clear whether the protons coming from the antisunward direction (relative to the electron strahl) represent a partial mirroring of the beam from the sunward direction, or a substantially reduced injection and acceleration of particles coming from this direction. In any event, the anisotropy diminished from $\sim 15: 00$ UT on 18 August until $\sim$ 01:00 UT on 19 August (Figure 8) as the intensities from the sunward direction steadily decreased while those from the antisunward direction and perpendicular to the field increased (Figure 5); the overall intensity remained essentially constant or increased slightly (Figure 2). As the shock that accelerated the particles moved outward through the magnetic cloud, the ambient magnetic field would have decreased (as would the magnetic field gradient between the shock and the spacecraft), which would have resulted in less magnetic focusing and thus reduced anisotropy.

At the back end of the magnetic cloud, from 01:00 UT to 05:00 UT on 19 August, the LET protons became essentially isotropic (such a period of greatly reduced anisotropies was also present within the magnetic cloud that generated the large anisotropies in the May 1998 event discussed by Torsti, Riihonen, and Kocharov, 2004). Although this coincides with an excursion of the $B$ field latitude to directions outside of LET's field of view (Figure 4), this does not appear to be an artifact of limited pitch angle coverage, as the intensity distribution in the pitch angles that are sampled also shows a clear change at this time (Figure 8). Furthermore, the electron strahl measured by SWEA simultaneously showed a pronounced broadening, indicating an increase in particle scattering (or a decrease in focusing). This period also featured a number of other unusual signatures, such as an abrupt drop in $\mathrm{He} / \mathrm{H}$ by a factor of four at $1.8-3.6 \mathrm{MeV} /$ nucleon and a slight hardening of the proton spectrum (Figure 9). One might want to interpret this as an indication of a new injection of particles from a different SEP event with a lower $\mathrm{He} / \mathrm{H}$ ratio, but there was no increase in the proton intensities at this time (Figure 2); the decrease in $\mathrm{He} / \mathrm{H}$ is entirely due to a sudden drop in the He intensity. The decrease is smaller at higher energies, or equivalently, the energy dependence of the $\mathrm{He} / \mathrm{H}$ ratio is much smaller at this time than during the surrounding periods of the SEP event.

Velocity dispersion browse plots from SIT show an apparent decrease in intensity during this same isotropic period (http://www.srl.caltech.edu/STEREO/Level1/SIT_public.html). This decrease is reminiscent of the intermittent intensity depletions reported in small, impulsive events (Mazur et al., 2000) that arise from intertwining of magnetic flux tubes that contain or do not contain energetic particles, depending on whether or not they are connected to the particle source at the Sun. Even the four-hour duration of this isotropic period is consistent with the average 3.2-hour duration found for the intensity dropouts (Mazur et al., 2000), but it is unusual to find such dropouts in large events. Furthermore, the dropouts here seem to exhibit a dependence on species or energy, with a general depletion of particles with mass greater than ten amu at energies above $400 \mathrm{keV} /$ nucleon in SIT, while in LET the depletions in $\mathrm{He}$ are more pronounced at energies below $\sim 5 \mathrm{MeV} /$ nucleon (Figure 9) and 
absent in $\mathrm{H}$ for all energies above 1.8 MeV (Figure 2). Other observations in the solar wind have found significant compositional variability within CME ejecta (Lepri and Zurbuchen, 2010); if such variability was also present in the seed material for the energetic particles, it might contribute to the unusual signatures in this cloud. Clearly, more analysis and modeling are necessary to understand this unusual period.

From the end of the isotropic period at $\sim$ 05:00 UT on 19 August until the shock arrival at 16:14 UT on 20 August, the directions of the energetic proton and suprathermal electron anisotropies remained generally similar to each other (Figure 8). Flow directions (relative to the magnetic field polarity) of both species reversed several times on 20 August (e.g., compare the directions at 06:00 UT and 10:00 UT with those at 08:00 UT in Figure 8) at magnetic sector boundary crossings (Figure 4). However, after the shock passage, the small anisotropy remaining in the energetic proton pitch angle distributions was directed opposite to that of the suprathermal electrons (Figure 8). That is, protons downstream of the shock were flowing back toward the Sun, as expected if the shock were the source of the energetic particles and has been reported in other events (e.g., von Rosenvinge and Reames, 1983).

Several hours before the arrival of the strong shock on 20 August, a series of highly anisotropic shock spikes was observed (Figure 5). This type of spike generally has a high intensity only when the shock normal is almost perpendicular to the magnetic field (Sarris and Van Allen, 1974). A a series of spikes may possibly be created when fluctuations in the field direction or ripples in the shock surface cause the local geometry to be more nearly perpendicular, with acceleration ceasing and intensities falling whenever the field becomes more parallel to the shock (Sarris and Van Allen, 1974). At the upstream spikes, the proton spectra became softer, and the He/H ratio dropped (Figure 9). However, the spike at the shock itself had a harder proton spectrum and an increase in $\mathrm{He} / \mathrm{H}$; this behavior is rather unusual and warrants further study. The maximum particle intensity is expected to be a few gyroradii downstream from the shock front (Sarris and Van Allen, 1974), so the intensities of higher energy particles should peak later, as observed (Figure 10).

\section{Conclusions}

Particles in the 18 August 2010 event appear to have been injected into a magnetic cloud, where they could travel along this conduit with little scattering (due to the less turbulent fields), and become highly anisotropic through magnetic focusing; their bidirectional flow indicates either injection at both footpoints of the cloud or magnetic mirroring. Many such events have been studied (e.g., Marsden et al., 1987; Richardson, Cane, and von Rosenvinge, 1991; Richardson et al., 2000), but rarely have such large anisotropies been reported; one previous example was the May 1998 event observed by SOHO (Torsti, Riihonen, and Kocharov, 2004). Also, the combination of all the features observed in this one event seems unusual. For example, in the survey of Richardson and Cane (1996), of 39 SEP events that occurred when the observing spacecraft was located in preexisting ejecta, only one or two exhibited all four of the following: i) low proton temperatures, ii) magnetic cloud signatures, iii) bidirectional solar wind heat flux, and iv) flows of bidirectional ions at energies $>1 \mathrm{MeV}$. All of these characteristics (and more) were apparent in the 18 August 2010 event. Thus, this event provides an interesting case study for energetic particle transport in the heliosphere under unusual conditions.

The wealth of detailed particle data presented here could be used to test particle transport models. Preliminary modeling of this event (Gómez-Herrero et al., 2011) attempts to reproduce the electron time profiles over the broad range of longitudes at which they were 
observed. While perhaps adequate to account for the electron event onset (which took place before the arrival of the magnetic cloud; see Figure 3), this model at present does not include the magnetic cloud, which seems to be required to account for the anisotropies of the energetic protons reported here.

Acknowledgements This work was supported by the National Aeronautics and Space Administration (NASA) at Caltech and JPL under subcontract SA2715-26309 from the University of California at Berkeley under NASA contract NAS5-03131, and by NASA award NNX08AK87G. We thank the PLASTIC (NASA contract NAS5-00132), MAG, and SWEA investigators on STEREO for making their data publicly available. This work benefited greatly from discussions at "The Sun-360 Workshop" held at the Christian-AlbrechtsUniversität in Kiel, Germany in July 2011. We thank B. Dotson for help in analyzing the data and in preparing the figures.

\section{References}

Acuña, M.H., Curtis, D., Scheifele, J.L., Russell, C.T., Schroeder, P., Szabo, A., Luhmann, J.: 2008, Space Sci. Rev. 136, 203.

Burlaga, L., Sittler, E., Mariani, F., Schwenn, R.: 1981, J. Geophys. Res. 86, 6673.

Cohen, C.M.S., Mason, G.M., Haggerty, D.K., Gómez-Herrero, R., von Rosenvinge, T.T., Mewaldt, R.A., et al.: 2010, In: AGU Fall. Meeting abstract SH33B-1844.

Crooker, N.U., Kahler, S.W., Larson, D.E., Lin, R.P.: 2004, J. Geophys. Res. 109, A03108. doi:10.1029/2003/JA010278.

Elliott, H.A., McComas, D.J., Schwadron, N.A., Gosling, J.T., Skoug, R.M., Gloeckler, G., Zurbuchen, T.H.: 2005, J. Geophys. Res. 110, A04103. doi:10.1029/2004JA010794.

Galvin, A.B., Kistler, L.M., Popecki, M.A., Farrugia, C.J., Simunac, K.D.C., Ellis, L., et al.: 2008, Space Sci. Rev. 136, 437.

Giacalone, J., Kóta, J.: 2006, Space Sci. Rev. 124, 277.

Giacalone, J., Jokipii, J.R.: 2006, J. Phys. Conf. Ser. 47, 160.

Gómez-Herrero, R., Kartavykh, Y., Dröge, W., Klassen, A., Dresing, N., Klecker, B., et al.: 2011, In: Proc. 32nd Internat. Cosmic Ray Conf. (Beijing) 10, Institute of High Energy Physics, Beijing, 201 - 203.

Gosling, J.T., Baker, D.N., Bame, S.J., Feldman, W.C., Zwickl, R.D.: 1987, J. Geophys. Res. $92,8519$.

Haggerty, D.K., Roelof, E.C.: 2002, Astrophys. J. 579, 841.

Ipavich, F.M.: 1974, Geophys. Res. Lett. 1, 149.

Kahler, S., Haggerty, D.K., Richardson, I.G.: 2011, Astrophys. J. 736, 106.

Kahler, S., Ragot, B.R.: 2006, Astrophys. J. 646, 634.

Krucker, S., Lin, R.P.: 2000, Astrophys. J. Lett. 542, L61.

Larson, D.E., Lin, R.P., McTiernan, J.M., McFadden, J.P., Ergun, R.E., McCarthy, M., et al.: 1997, Geophys. Res. Lett. 24, 1911.

Lepri, S.T., Zurbuchen, T.H.: 2010, Astrophys. J. 731, L22. doi:10.1088/2041-8205/723/1/L22.

Leske, R.A., Cohen, C.M.S., Mewaldt, R.A., Cummings, A.C., Stone, E.C., Wiedenbeck, M.E., von Rosenvinge, T.T.: 2011, In: Proc. 32nd Internat. Cosmic Ray Conf. (Beijing) 10, Institute of High Energy Physics, Beijing, 197.

Lin, R.P., Potter, D.W., Gurnett, D.A., Scarf, F.L.: 1981, Astrophys. J. 251, 364.

Lintunen, J., Vainio, R.: 2004, Astron. Astrophys. 420, 343.

Marsden, R.G., Sanderson, T.R., Tranquille, C., Wenzel, K.-P.: 1987, J. Geophys. Res. 92, 11009.

Mason, G.M., Korth, A., Walpole, P.H., Desai, M.I., von Rosenvinge, T.T., Shuman, S.A.: 2008, Space Sci. Rev. 136, 257.

Mazur, J.E., Mason, G.M., Dwyer, J.R., Giacalone, J., Jokipii, J.R., Stone, E.C.: 2000, Astrophys. J. 532, L79.

Mewaldt, R.A., Cohen, C.M.S., Labrador, A.W., Leske, R.A., Mason, G.M., Desai, M.I., et al.: 2005, J. Geophys. Res. 110, A09S18. doi:10.1029/2005JA011038.

Mewaldt, R.A., Cohen, C.M.S., Cook, W.R., Cummings, A.C., Davis, A.J., Geier, S., et al.: 2008, Space Sci. Rev. 136, 285.

Müller-Mellin, R., Böttcher, S., Falenski, J., Rode, E., Duvet, L., Sanderson, T., et al.: 2008, Space Sci. Rev. 136, 363.

Reames, D.V.: 1995, Adv. Space Res. 15(7), 41.

Richardson, I.G., Cane, H.V.: 1996, J. Geophys. Res. 101, 27521.

Richardson, I.G., Cane, H.V., von Rosenvinge, T.T.: 1991, J. Geophys. Res. 96, 7853. 
Richardson, I.G., Dvornikov, V.M., Sdobnov, V.E., Cane, H.V.: 2000, J. Geophys. Res. 105, 12579.

Sáiz, A., Evenson, P., Ruffolo, D., Bieber, J.W.: 2005, Astrophys. J. 626, 1131.

Sarris, E.T., Van Allen, J.A.: 1974, J. Geophys. Res. 79, 4157.

Sauvaud, J.-A., Larson, D., Aoustin, C., Curtis, D., Médale, J.-L., Federov, A., et al.: 2008, Space Sci. Rev. 136, 227.

Sonnerup, B.U.Ö., Cahill, L.J. Jr.: 1967, J. Geophys. Res. 72, 171.

Steed, K., Lapenta, G.: 2011, In: AGU Fall Meeting Abstract, SH23C-1974.

Steed, K., Owen, C.J., Démoulin, P., Dasso, S.: 2011, J. Geophys. Res. 116, A01106. doi:10.1029/2010JA015940.

Torsti, J., Riihonen, E., Kocharov, L.: 2004, Astrophys. J. 600, L83.

von Rosenvinge, T.T., Reames, D.V.: 1983, In: Proc. 18th Internat. Cosmic Ray Conf. (Bangalore) 10, Tata Institute of Fundamental Research, Bombay p. 373.

von Rosenvinge, T.T., Reames, D.V., Baker, R., Hawk, J., Nolan, J.T., Ryan, L., et al.: 2008, Space Sci. Rev. 136, 391. 